\title{
Predictors of Viral Load Test Result over Time among HIV Adult Patients on Highly Active Antiretroviral Therapy (HAART): A Retrospective Study in Zewditu Memorial Hospital, Ethiopia
}

Ayitenew Agegn ( $\nabla$ aytage059@gmail.com )

Dire Dawa University

Mengistu Abebe

Dire Dawa University

Kidist Belay

Dire Dawa University

Article

Keywords: logistic regression, HAART, retrospective, generalized linear mixed model, odds ratio

Posted Date: May 5th, 2022

DOI: https://doi.org/10.21203/rs.3.rs-1361144/v2

License: (c) (1) This work is licensed under a Creative Commons Attribution 4.0 International License.

Read Full License 


\section{Abstract}

Background: HIV attacks the CD4 cell which is responsible for the body's immune response to infectious agents. The main objective of this study was to identify Predictors of Viral Load Result over time among HIV Patients Initiated HAART in Zewditu Memorial Hospital.

Methods: A retrospective cohort study design was conducted on 161 HIV infected patients under HAART whose follow-ups were from January 2014 up to December 2017. Generalized linear mixed-effects model (Binary logistic regression model) for status of viral load result was used for inferential data analysis. The Hosmer and Lemeshow Goodness of fit Test and the likelihood ratio tests were used for measures of goodness of fit for categorical data

Results: The descriptive statistics revealed that the longitudinal response variable which was viral load status among HIV patient's, in the study 90 of the patients were detected at baseline and 71 of them were not detected at baseline measurements. The estimated odds of the detected patients who lived in rural area was found to be about $6.3(\mathrm{AOR}=6.30)$ times that of the odds of detected patients in urban area controlling for the other variables in the model. The estimated odds of the detected disclosed patients was found to be about $80.5 \%$ ( $A O R=0.195$ ) less likely than the odds of detected not disclosed Patients by controlling for the other variables in the model.

Conclusions: The predictor variables; observation time, weight, residence, age, educational level, clinical stage, functional status, baseline CD 4 cell count and disclosure were statistically and significantly associated with status of viral load. The proportional odds model assumption was also checked numerically using Hosmer and Lemeshow goodness-of-fit statistics. The results justified that the assumption was fulfilled.

\section{Introduction}

HIV (Human Immune Deficiency Virus) is a virus that causes Acquired Immune deficiency Syndrome (AIDS) by reducing a person's ability to fight infection. HIV attacks an immune cell called the CD4 cell which is responsible for the body's immune response to infectious agents. This CD4 cells, decrease in number with time from the HIV virus, so that an infected person's CD4cell count can be used to monitor the progression of the disease (1). HIV results in increased susceptibility to a wide range of infections, cancers and other diseases that people with healthy immune systems can fight off. According to UNAIDS fact sheet updated report, 36.7 million people were living with HIV globally in 2016. Among those infected people adult's account for 34.5 million. Since the start of the epidemic in 1981, 76.1 million people have become infected by the virus and 35.0 million people have died from AIDS related illness respectively (15).

In 2016, there were 25.6 million peoples living with HIV in Africa, from these $54 \%$ of people were receiving antiretroviral treatment (15). At the end of 2016, there were 19.4 million people living with HIV in eastern and southern Africa. Women and girls account for more than half (59\%) of the total number of people 
living with HIV. In Africa, the rate of viral suppression among PLWH in the Eastern and Southern regions are reported to be $52 \%$ (15). Not with standing, with additional 4.0 million PLWH recorded in 2017, the Eastern and Southern Africa countries could be confronted with enormous challenge to achieving the third 90 (4). North, Central, and West Africa are far behind with an estimated proportion of $29 \%$ who achieved viral suppression in 2017 (15). Recent national statistics from the Ethiopia AIDS Commission (GAC) show that the country achieved $55.3 \%$ in the first $90,61.2 \%$ in the second 90 , and $64.3 \%$ in the third 90 (4). Attaining viral load suppression is very necessary for controlling vertical and horizontal transmission of the virus within the population (5).

As a result of recent advances ART introduced in 1996, HIV-positive people now live and these effective antiretroviral drugs can control the virus and help to prevent transmission so that people with HIV and those at substantial risk, can enjoy healthy, long and productive lives (14). ART was introduced in Ethiopia in 2003, and in 2005 the Ethiopian government launched free access in different health sectors to improve quality of life of PLWHA (13). Even though the number of people living with HIV on antiretroviral treatment is increasing from year to year, there are a number of AIDS-related deaths registered every year. This indicates that there are other factors affecting the progression of the disease and the survival status of HIV positive patients as well. Therefore, it is important to asses other factors those affect the effectiveness of the treatment over time. This can be done by measuring viral load results over time. Most researches conducted in relation to HIV infected patients are on the survival time of the patients after the initiation of ART (7), (9). There were researches conducted in relation with survival time of the patients after the initiation of ART and level of ART adherence in Debre Marko's Referral Hospital (11), (10). But there was no enough evidence that researches have been conducted on the viral load results of HIV-infected patients who are under the treatment of antiretroviral therapy in the Hospital. Therefore, the main objective of current investigation was Predictors of Viral Load Test Result over Time among HIV Adult Patients on Highly Active Antiretroviral Therapy (HAART): A Retrospective Study in Zewditu Memorial Hospital, Ethiopia.

The result of the study may enable clinicians to enhance the awareness of the society about factors that increase the probability of death by HIV/AIDS. It helps the government \& non-governmental institutions to take evidence-based interventions to give awareness for factors and covariates that affect HIV/AIDS and related disease. It recommends different methods to control the disease progression.

\section{Materials And Methods}

\section{Study Area and Design:}

A retrospective cohort study design was conducted at Zewditu Memorial Hospital, in Addis Abebe, Ethiopia.

\section{Source of data}


The HIV infected patients under HAART in the hospital were considered as source of population for this study. In this study, secondary data collected by health staff for treatment purpose were used for data analysis. The data was collected from the medical chart of each HIV/AIDS patient in the ART clinic in the hospital.

\section{Study population}

The study population consists of all HIV positive adult patients and started their HAART treatment in January, 2014 - December, 2017.

\section{Inclusion Criteria}

HIV/AIDS infected patients who had at least two follow ups in ART clinic for refilling their prescription and who were initiated their treatment during 1st January 2014 to 31 st December 2017 at Zewditu Memorial Hospital were eligible for current study.

\section{Sample size determination}

For current investigation, there were 1304 HIV positive patients whose follow ups were from January 2014 to December 2017. Among these patients, 161 were adult patients and considered as a sample. Hence, all patients under treatment whose follow ups were in the study period considered as sample size.

\section{Variables under current investigation}

The response variable under current investigation was viral load results of HIV positive patients under treatment. The viral load result was measured every six months. From this response, those who has detected (viral load result which is greater than or equal to 1000 cell/mm3) and undetected (viral load result which is less than 1000 cell/mm3) are classified under viral load test result of HIV infected patients (17). Thus the response variable of this study was denoted as

$$
Y=\left\{\begin{array}{c}
1, \text { detected) } \\
0, \text { undetected) }
\end{array}\right.
$$

The predictor (independent) covariates under current investigation were sex(male, female), weight in kg, residence area (rural, urban), age in years, marital status (living with partner, living without partner) ,education level( non-educated, educated), level of disclosure (yes, no), clinical stage( stage1, stage2, stage3, stage4), functional status (ambulatory, bedridden, working), level of adherence(adherent, nonadherent), TB co infection status (no, yes).

\section{Data Analysis}

Descriptive statistics are used to describe the basic features of the data in a study. They provide simple summaries about the sample and the measures. The collected data would be cleaned, edited and entered into the latest SPSS version 26. Frequency distributions, computing percentages, cross tabulations, mean 
and standard deviation, graphs and diagrams will be answered by descriptive statistics. Inferential statistics are used when a researcher wants to know how variables are related to one another and whether there is any difference between two or more groups. Inferential statistics allow one to make inferences from the sample to the population (8). The data were analyzed using SAS and R statistical software packages.

In data analysis, both fixed and random effects were included in the generalized linear mixed effect model. The fixed effect accounted for the set of predictors that are fixed across the subjects and described the relationships between predictor and response variables. The random-effect belongs to subject specific effects. In this study, we carried out by binary Logistic Regression Analysis.

\section{Binary Logistic Regression}

Logistic regression is a popular modeling approach when the dependent variable is dichotomous, ordinal or multinomial. It allows predicting the log odds of outcomes of a dependent variable from a set of variables that may be continuous, discrete, categorical, or a mix of any of these. Thus logistic regression is used in a wide range of applications leading to binary dependent data analysis (2). The error term in binary logistic regression is distributed binomially, not normally. Consequently, the response variable in logistic regression is not restricted to normality in case of parameter estimation. Because of this logistic regression is the most popular method of analyzing binary response data.

Covariance structures: Among the various covariance structures, compound symmetry (CS), unstructured (UN) and first order autoregressive (AR (1) were conducted and compared each other to identify the one which fitted the data well. During comparison of the three structures, AIC and BIC information criterion were considered provided that the one with smallest information criterion as the best for current data.

\section{Methods of Parameter Estimation}

Parameter estimation in current investigation was conducted using maximum likelihood (ML). The maximum likelihood estimator (MLE) can be obtained by maximizing the joint probability (likelihood function) for values of the data. Maximum likelihood estimation includes both regression coefficient and the variance components, that is, both fixed-effects and random effects terms in the likelihood function and it treats parameters as fixed but unknown quantities when the variance component is estimated. Method of parameter estimation for logistic model was conducted for the subject-specific and marginal residuals

\section{The Assessment of Goodness of Fit of Logistic Regression Model}

We begin our description of methods for assessing the fit of an estimated logistic regression model with the assumption that we are at least preliminarily satisfied with our efforts at the model building stage. By this we mean that, to the best of our knowledge, the model contains those variables (main effects as well as interactions) that should be in the model and that variables have been entered in the correct functional 
form. Now we would like to know how effectively the model describes the outcome variable. The goodness of fit measures how the model describes the response variable and it also investigating how close are predicted by the model with the observed values (3). The Hosmer and Lemeshow Goodness of fit Test and the likelihood ratio tests are the most commonly used measures of goodness of fit for categorical data (8) and Wald test statistic used to test the significance of individual parameters (coefficients).

\section{Missing Data Treatment}

Missing data (or missing values) is defined as the data value that is not stored for a variable in the observation of interest. There were different imputing missing values in the longitudinal study. The most popular imputation method for handling missing value is multiple imputations (12).

\section{Results}

Table 1 below displays patient's characteristics for the HIV/AIDS data from Zewditu memorial Hospital. Out of a sample of 161 patients, $67.7 \%$ were females and the remaining $32.3 \%$ were males. Majority of patients were from urban area $67.7 \%$ and $56.52 \%$ of the participants were employed, while $43.48 \%$ of patients had unemployment statuses were unemployed. When we see the educational level of patients, $68.32 \%$ were educated and the remaining $31.68 \%$ were none educated. Majority $(50.17 \%)$ of the infected patients were with working functional status, (i.e., an individual able to perform usual work in and out of the house), followed by those with ambulatory type of functional status who accounted for $30.21 \%$ of the total, and $19.62 \%$ were unable to perform activities of daily living (bedridden) patients. Regarding the clinical stage of patients, $17.39 \%$ were at clinical stage I, $32.30 \%$ at clinical stage II, $27.33 \%$ at clinical stage III and the rest $18.3 \%$ were at clinical stage IV when they started HAART. The adherence statuses of patients, $60.25 \%$ were good adherent, but $24.22 \%$ were fairly adhered and the remaining of patients was poorly adhered. At enrolment, the average baseline age in years, baselines CD 4 per $\mathrm{mm} 3$ and baseline weight per kg were $31.47,221$ and 48.46 respectively. 
Table 1

Baseline characteristics of potential predictors for HIV/AIDS patients

\begin{tabular}{|c|c|c|c|c|}
\hline \multirow[t]{2}{*}{ Characteristics } & \multirow[t]{2}{*}{ Category } & \multicolumn{2}{|c|}{ Status of Viral load } & \multirow{2}{*}{$\begin{array}{l}\text { Total } \\
(100 \%)\end{array}$} \\
\hline & & Detected (\%) & No detected (\%) & \\
\hline \multirow[t]{2}{*}{ Sex } & Male & $30(18.63)$ & $22(13.66)$ & $52(32.30)$ \\
\hline & Female & $58(36.02)$ & $51(31.64)$ & 109(67.7) \\
\hline \multirow[t]{2}{*}{ Marital status } & $\begin{array}{l}\text { Living with } \\
\text { partner }\end{array}$ & $46(28.57)$ & $57(35.40)$ & 103(63.97) \\
\hline & $\begin{array}{l}\text { Living } \\
\text { without } \\
\text { partner }\end{array}$ & $34(21.12)$ & $24(14.91)$ & $58(36.03)$ \\
\hline \multirow[t]{2}{*}{ Residence } & Urban & 72(44.72) & $37(22.98)$ & $109(67.70)$ \\
\hline & Rural & $27(16.77)$ & 25(15.53) & $52(32.30)$ \\
\hline \multirow{2}{*}{$\begin{array}{l}\text { Educational } \\
\text { level }\end{array}$} & Educated & 62(38.51) & $48(29.81)$ & $110(68.32)$ \\
\hline & Not educa.ted & $34(21.12)$ & 17(10.56) & $51(31.68)$ \\
\hline \multirow[t]{3}{*}{ Religion } & Muslim & $34(21.12)$ & 22(18.66) & $56(34.78)$ \\
\hline & Orthodox & $32(19.88)$ & $23(14.29)$ & $55(34.16)$ \\
\hline & Other & $31(19.25)$ & $19(11.80)$ & $50(31.06)$ \\
\hline \multirow[t]{2}{*}{ Occupation } & Employed & $43(26.71)$ & $48(29.81)$ & $91(56.52)$ \\
\hline & Unemployed & 29(18.01) & $41(25.47)$ & $70(43.48)$ \\
\hline
\end{tabular}




\begin{tabular}{|c|c|c|c|c|}
\hline \multirow[t]{4}{*}{ Clinical Stage } & Stage I & 18(11.18) & $10(6.21)$ & 28(17.39) \\
\hline & Stage II & $38(23.60)$ & $14(8.70)$ & $52(32.30)$ \\
\hline & Stage III & $23(14.29)$ & 21(13.04) & $44(27.33)$ \\
\hline & Stage IV & $22(13.66)$ & 15(9.4) & $37(18.3)$ \\
\hline \multirow[t]{3}{*}{ Functional status } & Ambulatory & $35(21.74)$ & 14(8.70) & $49(30.21)$ \\
\hline & Bedridden & 18(11.18) & $14(8.70)$ & 32(19.62) \\
\hline & Working & $54(27.33)$ & 26(13.04) & $80(50.17)$ \\
\hline \multirow[t]{2}{*}{ TB status } & Negative & 102(63.35) & 23(14.29) & 125(77.64) \\
\hline & Positive & 28(17.39) & $8(4.97)$ & $36(22.36)$ \\
\hline \multirow[t]{3}{*}{ Adherence } & Poor & $17(10.56)$ & $8(4.97)$ & $25(15.53)$ \\
\hline & Fair & $24(14.91)$ & 15(9.32) & $39(24.22)$ \\
\hline & Good & 79(49.07) & $18(11.18)$ & $97(60.25)$ \\
\hline \multicolumn{5}{|l|}{ Continuous covariates } \\
\hline Average baseline age in years(std.dev) & & $31.47(7.62)$ & & \\
\hline average baseline CD4/mm3 (std.dev) & & $221(156)$ & & \\
\hline Average baseline weight/kg (std.dev) & & $48.46(12.58)$ & & \\
\hline
\end{tabular}

Table 2 below shows that the longitudinal response variable which was viral load status among HIV patient's, in the study 90 of the patients were detected at baseline, $6^{\text {th }}$ and $12^{\text {th }}$ measurements and 71 of them were not detected at baseline, $6^{\text {th }}$ and $12^{\text {th }}$ measurements.

Table 2

summaries of descriptive statistics for the response variables

\begin{tabular}{|llllllll|}
\hline Time & & $\mathbf{0}$ & $\mathbf{6}$ & $\mathbf{1 2}$ & $\mathbf{1 8}$ & $\mathbf{2 4}$ & $\mathbf{3 0}$ \\
\hline Status of viral load & Detected & 90 & 90 & 90 & 73 & 48 & 39 \\
& Not detected & 71 & 71 & 71 & 57 & 40 & 30 \\
\hline
\end{tabular}

\section{Selection of Covariance Structure in a Generalized Linear Mixed Model}

For GLMM to be valid, the covariance among repeated measures must be modeled properly. 
To identify the appropriate covariance structure, the commonly used covariance structures which are compound symmetry (CS), first-order autoregressive (AR (1)), heterogeneous compound symmetry (CSH), heterogeneous first-order autoregressive (HAR (1)), and unstructured (UN) were considered

Table 3

Comparison of covariance structure for generalized linear mixed-effects model

\begin{tabular}{|lll|}
\hline Covariance structure & AIC & BIC \\
\hline CS & 171131.4 & 171214.1 \\
\hline AR (1) & 172152.3 & 172234.2 \\
\hline UN & 154412.9 & 154497.9 \\
\hline ARH (1) & 162152.3 & 162234.2 \\
CSH & 182131.4 & 182214.1 \\
\hline
\end{tabular}

In above Table 3 the smallest values of AIC and BIC for the unstructured (UN) model suggest that this unstructured covariance structure (UN) was the best fit to our data as compared to the remaining covariance structures.

\section{Selection of Random Effects in a Generalized Linear Mixed Model}

Based on unstructured (UN) covariance structure we implemented different generalized linear mixed models to study the longitudinal outcome by including the subject-specific random effects. Finally, we compared the information criteria values for the selection of random effects to be included in the generalized linear mixed-effects model.

Table 4

Selection of random effects to be included in the GLMM

\begin{tabular}{|lll|}
\hline Models for the random effect & AIC & BIC \\
\hline Random intercept & 172150.3 & 172229.0 \\
\hline Random slope & 15751.58 & 15877.20 \\
\hline Random intercept and slope & 143412.9 & 143497.9 \\
\hline
\end{tabular}

According to above Table 4, we can choose the random intercept and slope model which allows the intercept and slope to vary randomly among individuals. That means individual status of viral load of HIV patients vary from visit to visit randomly. Therefore, the random intercept and slope model is a more 
parsimonious model for the generalized linear mixed-effects model based on its smaller values of AIC and BIC.

\section{Multivariable Logistic Regression model}

Multivariable analysis of linear mixed model was done by using all significant covariates at univariable analysis. Below Table 5 displays the result of final generalized linear mixed model, the result showed that the predictor Obstime, Age, Weight, BaselineCD4, Residence, Clinical stage, Functional status and Disclosure were statistically significant factors of status of viral load result at a $5 \%$ level of significance.

According to the data set we considered the generalized linear mixed model (both fixed and random effect), to study the longitudinal outcome by including the subject-specific random effects. And we compared random intercept and random slope to fit the appropriate model. 
Table 5

Parameter Estimates, Standard Errors (Std.Err), 95\% Cl and OR under the generalized linear-mixed effects analysis

\begin{tabular}{|c|c|c|c|c|}
\hline Covariates & Estimate & Std.Err & OR (Cl (95\%)) & p-value \\
\hline Intercepts & 5.0850 & 1.767 & $161.60(4.97,5261.6)$ & 0.002 \\
\hline Obstime & -.313586 & .073 & $0.731(0.634,0.842)$ & 0.001 \\
\hline Age & -2.8645 & .040 & $0.057(0.0527,0.0617)$ & 0.001 \\
\hline Weight & -0.101 & 0.024 & $0.904(0.862,0.946)$ & 0.0001 \\
\hline BaselineCD4 & -0.004 & 0.001 & $0.996(0.994,0.998)$ & 0.0001 \\
\hline \multicolumn{5}{|c|}{$\begin{array}{l}\text { Education level(ref = No } \\
\text { education) }\end{array}$} \\
\hline Education & -3.505 & 1.638 & $0.030(0.002,0.385)$ & 0.0053 \\
\hline \multicolumn{5}{|c|}{ Residence (ref = urban ) } \\
\hline Rural & 1.840 & 0.645 & $6.30(1.78,22.25)$ & 0.0043 \\
\hline \multicolumn{5}{|c|}{ Clinical stage (ref = stage IV) } \\
\hline Stage I & -7.896 & 0.728 & $0.0004(0.0001,0.002)$ & 0.0001 \\
\hline Stage II & -7.550 & 0.694 & $0.0005(0.0001,0.002)$ & 0.0001 \\
\hline Stage III & -3.729 & 0.667 & $0.024(0.006,0.089)$ & 0.0001 \\
\hline \multicolumn{5}{|c|}{ Functional status $($ ref $=$ Ambulatory } \\
\hline Working & -.527 & .039 & $0.5905(0.547,0.638)$ & 0.001 \\
\hline Bedridden & .183 & .0755 & $1.199(1.035,1.391)$ & 0.016 \\
\hline \multicolumn{5}{|c|}{ Adherence $($ ref = Good $)$} \\
\hline Poor & .182 .0754131 & .0754 & $1.120(1.035,1.391)$ & 0.016 \\
\hline Fair & $\begin{array}{l}.047 \\
.0796088\end{array}$ & .0796 & $1.0477(0.897,1.225)$ & 0.558 \\
\hline \multicolumn{5}{|c|}{ Disclosure $($ ref $=$ no $)$} \\
\hline Yes & -1.635 & 0.593 & $0.195(0.023,0.818)$ & 0.0134 \\
\hline Random effects & Estimates & $95 \% \mathrm{Cl}$ & & \\
\hline \multicolumn{5}{|c|}{ Note: ref $=$ reference category of the categorical variable. } \\
\hline $\begin{array}{l}\text { Note: } b_{0 i} \text { and } b_{1 i} \\
\text { longitudinal sepa }\end{array}$ & in the random int & cept and & ndom slopes model for & \\
\hline
\end{tabular}




\begin{tabular}{|c|c|c|c|c|}
\hline Covariates & Estimate & Std.Err & OR (Cl (95\%)) & p-value \\
\hline & & Lower & Upper & \\
\hline Intercept $\left(S t d v\left(b_{0 i}\right)\right)$ & 2.6610 & 2.304 & 3.0379 & \\
\hline Obstime $\left(\operatorname{Std} v\left(b_{1 i}\right)\right)$ & 0.1884 & 0.1633 & 0.2245 & \\
\hline $\operatorname{cor}\left(b_{0 i}, b_{1 i}\right)$ & -0.0916 & -0.0802 & -0.1063 & \\
\hline \multicolumn{5}{|c|}{ Note: ref $=$ reference category of the categorical variable. } \\
\hline \multicolumn{5}{|c|}{$\begin{array}{l}\text { Note: } b_{0 i} \text { and } b_{1 i} \text { are random effects in the random intercept and random slopes model for } \\
\text { longitudinal separate model. }\end{array}$} \\
\hline
\end{tabular}

When the proportional odds model is used in the analysis of binary data, the coefficients of the explanatory variables in the model is interpreted as logarithm of the ratio of the odds of response variable. This means that estimates of this odds ratio, and corresponding confidence intervals, can be easily found from the fitted model. The interpretation of parameters corresponding to different variables which are found significant in the final model is described in the following section and Comparison is made with the reference category.

The estimated odds of the detected patients (viral load result which is greater than $1000 \mathrm{cell} / \mathrm{mm} 3$ ) who lived in Rural were found to be about $(\mathrm{OR}=6.30)$ times that of the odds of detected Patients (viral load result which is greater than $1000 \mathrm{cell} / \mathrm{mm} 3$ ) in urban controlling for the other variables in the model. The estimated odds of the detected patients (viral load result which is greater than $1000 \mathrm{cell} / \mathrm{mm} 3$ ) who have education were found to be about $(\mathrm{OR}=0.03)$ times that of the odds of detected Patients (viral load result which is greater than 1000 cell/mm3) who have no education by controlling for the other variables in the model. The estimated odds of the detected disclosed patients (viral load result which is greater than 1000 cell $/ \mathrm{mm} 3)$ were found to be about $80.5 \%(\mathrm{OR}=0.195)$ less likely than the odds of detected not disclosed Patients (viral load result which is greater than 1000 cell/mm3) by controlling for the other variables in the model. The estimated odds of the detected patients who have working functional status were found to be about $(\mathrm{OR}=0.5905)$ times that of the odds of detected Patients who have ambulatory functional status by controlling for the other variables in the model. The estimated odds of the detected patients who have clinical stage I was found to be about $(O R=0.0004)$ times that of the odds of detected Patients who have clinical stage IV by controlling for the other variables in the model. The estimated odds of the detected patients who have clinical stage II were found to be about $(\mathrm{OR}=0.0005)$ times that of the odds of detected Patients who have clinical stage IV by controlling for the other variables in the model.

\section{Assessment of Goodness of Fit of the Model}

In order to check the goodness-of-fit of an estimated binary logistic regression model one should assume that the model contains those variables that should be in the model and have been entered in the correct functional form. 


\section{Hosmer and Lemeshow Test}

Hosmer and Lemeshow goodness-of-fit statistic measure the correspondence between the actual and predicted values of the dependent variable. From the Table 6 below, Hosmer and Lemeshow Test with $p$ value (0.0854) are greater than 0.05 since we do not reject the null hypothesis. Therefore, the model is good fit. It indicates that binary logistic regression model of status of viral load result fits the data very well.

Table 6

Hosmer and Lemeshow test result

\begin{tabular}{|llll|}
\hline Test & Chi-Square & DF & p-value \\
\hline Hosmer and Lemeshow test & 14.795 & 8 & 0.0854 \\
\hline
\end{tabular}

\section{Discussion}

The results from this retrospective study, the main effects like education level, clinical stage, functional status and adherence had significant effect on viral load result. A status of viral load was found evolving differently between educated and non-educated patients. This might occur because as patients become more educated, they may have better care of their health and they may have enough understanding about HAART and further leads the status of viral load may not detected and leads to live for long period of time(5).

WHO clinical stages have significant effects for the status of viral load result and weight of patients. Hence, patients who started their treatment at earlier stage can survive for long period of time because of good recovery of the viral load result (4). This might make them able to take the treatment properly due to less replication of the virus in their body (4).

Functional status of the HIV patients affects the status of viral load result. Hence, patients at bedridden are at high risk for $d$ recovery of viral load result. On the other hand, Patients who are in working functional status can take prescribed medication by themselves on the time given by the health staff and leads for good recover of viral load result. Such patients can express their feeling for the health staff and this helps for the right direction/ decision of the health staff for ART program (4).

Adherences to prescribed medication have significant association for the variation of viral load result. Hence, patients who adhered the prescribed medication can recover their viral load result and they may have long live with the virus given the other external conditions constant. This result is supported by previously conducted researches (5)

\section{Conclusion}


The study examined the socio-demographic and clinical factors of viral load results of HIV/AIDS patients in Zewditu memorial hospital. Results of proportional odds model shows that socio-demographic and clinical variables are very important for determinant of status of viral load. The findings of the study show that different factors such as patient's age, educational level, employment status, marital status, adherence level ,WHO clinical stage and disclosure of their disease have statistically significant effect on the outcome of the variable. The proportional odds model assumption was also checked numerically using Hosmer and Lemeshow goodness-of-fit statistics. The results justified that the assumption was fulfilled.

\section{Recommendations}

From the results of our final model, we observed that being at later WHO stage (e.g. stage IV) has positive impact on status of viral load result. Therefore, patients with such characteristics need special guidance and due attention to improve their response to the treatment. This study was restricted only on some covariates. So, it is recommended that further studies of this nature include other important independent variables that were not included in this study such as, opportunistic infections, etc. Therefore, it is also recommended to consider time dependent covariates and use statistical methods that can handle missingness in the data. Finally, all government and non-government body should focus on developing and well integrated alternative income generating activities to ART programs for PLWHA to improve their nutritional status for those patients retention on ART treatment and to reduce viral load test results and burden of HIV/ADIS on society.

\section{Abbreviations}

HAART = Highly Active Anti-retroviral Therapy, PLWHA= People Living with HIV, FMOH = Federal Ministry of Health, HIV= Human Immunodeficiency Virus, $\mathrm{Cl}=$ Confidence Interval, GLMM= Generalized Linear Mixed effect Model, CD4 = Cluster Differentiation 4

\section{Declarations}

\section{Ethics approval and consent to participate}

The data was collected previously by the health staff for providing treatment/ to start HAART. The study was approved by the committees namely Dire Dawa University's Ethical Approval committee, Dire Dawa University, Ethiopia with reference number, Ref $\neq$ RCS/1456/2021. All patients provided written informed consent. Informed consent was given by ethical approved committee, and all have done by the guidelines of the institution

Consent for publication: Not applicable. 
Availability of data and materials: The data used for current investigation is available with hands of corresponding author.

Competing interests: No conflict of interest between authors and between authors and institutions.

Funding: It is not applicable

Author's contributions: $A A$ wrote the proposal, develop data collection format, supervise the data collection process, analyzed and interpreted the data. MA and KB participated in design and data analysis and critically read the manuscript and gave constructive comments for betterment of the manuscript based on her field of specialization.

Acknowledgement: We would like to express our profound gratitude to the management of Zewditu Memorial Hospital, for allowing us to have access to the pertinent medical registers from which we extracted the data used in this study.

\section{Author's information:}

1*Department of Statistics, College of Natural and Computational Sciences, Dire Dawa University, Dire Dawa, Ethiopia.

E-mail:aytage059@gmail.com

2*Department of Statistics, College of Natural and Computational Sciences, Dire Dawa University, Dire Dawa, Ethiopia.

E-mail:menge23a@gmail.com

$3{ }^{*}$ Department of Statistics, College of Natural and Computational Sciences, Dire Dawa University, Dire Dawa, Ethiopia.

E-mail:Kid21belay@gmail.com

\section{References}

1. Adams M, Luguterah A. Longitudinal analysis of change in CD 4 + cell counts of HIV-1 patients on antiretroviral therapy (ART) in the Builsa district hospital. European Scientific Journal. 2013;9(33).

2. Cheng PE, Liou J-W, Kao H-W, Liou M. A Constructive Procedure for Modeling Categorical Variables: Log-Linear and Logit Models. arXiv preprint arXiv:180101278. 2018.

3. Andersen EB. A goodness of fit test for the Rasch model. Psychometrika. 1973;38(1):123-40.

4. Jiamsakul A, Kariminia A, Althoff KN, Cesar C, Cortes CP, Davies M-A, et al. HIV viral load suppression in adults and children receiving antiretroviral therapy-results from the leDEA collaboration. Journal of acquired immune deficiency syndromes (1999). 2017;76(3):319. 
5. Mujugira A, Celum C, Tappero JW, Ronald A, Mugo N, Baeten JM. Younger age predicts failure to achieve viral suppression and virologic rebound among HIV-1-infected persons in serodiscordant partnerships. AIDS research and human retroviruses. 2016;32(2):148-54.

6. Bhatta L, Klouman E, Deuba K, Shrestha R, Karki DK, Ekstrom AM, et al. Survival on antiretroviral treatment among adult HIV-infected patients in Nepal: a retrospective cohort study in far-western Region, 2006-2011. BMC infectious diseases. 2013;13(1):1-9.

7. Chakravarty J, Tiwary NK, Prasad SR, Shukla S, Tiwari A, Mishra RN, et al. Determinants of survival in adult HIV patients on antiretroviral therapy in Eastern Uttar Pradesh: a prospective study. The Indian Journal of Medical Research. 2014;140(4):491.

8. Hosmer Jr DW, Lemeshow S, Sturdivant RX. Applied logistic regression: John Wiley \& Sons; 2013.

9. Ayalew J, Moges $\mathrm{H}$, Worku A. Identifying factors related to the survival of AIDS patients under the follow-up of antiretroviral therapy (ART): the case of South Wollo. Int J Data Envelopment Anal Oper Res. 2014;1:21-7.

10. Asmare M, Aychiluhem M, Ayana M, Jara D. Level of ART adherence and associated factors among HIV sero-positive adult on highly active antiretroviral therapy in Debre Markos Referral Hospital, Northwest Ethiopia. J Antivir Antiretrovir. 2014;6(3):120-6.

11. Abebe N, Alemu K, Asfaw T, Abajobir AA. Predictors of mortality among HIV positive adults on antiretroviral therapy in Debremarkos Referral Hospital, Northwest Ethiopia. Journal of AIDS and HIV Research. 2014;6(1):19-27.

12. Singer JD, Willett JB, Willett JB. Applied longitudinal data analysis: Modeling change and event occurrence: Oxford university press; 2003.

13. Tsegaye AT, Wubshet M, Awoke T, Alene KA. Predictors of treatment failure on second-line antiretroviral therapy among adults in northwest Ethiopia: a multicentre retrospective follow-up study. BMJ open. 2016;6(12):e012537.

14. UNAIDS. Fact sheet on HIV/AIDS,World AIDS Day. 2016

15. Unaids J. Fact sheet-latest global and regional statistics on the status of the AIDS epidemic. Geneva: UNAIDS. 2017.

16. Wang H, Wolock TM, Carter A, Nguyen G, Kyu HH, Gakidou E, et al. Estimates of global, regional, and national incidence, prevalence, and mortality of HIV, 1980-2015: the Global Burden of Disease Study 2015. The lancet HIV. 2016;3(8):e361-e87.

17. Webmd.'HIV Viral Load: Types of Tests, What Do Results Mean CORONAVIRUS', pp. 2020: 1-4. 